\title{
Stochastic deletion-insertion algorithm to construct dense linkage maps
}

\author{
Jixiang Wu* WiAng-Yang Lou $^{\ddagger}$ And Michael Gonda*
}

In this study, we proposed a stochastic deletion-insertion (SDI) algorithm for constructing large-scale linkage maps. This SDI algorithm was compared with three published approximation approaches, the seriation (SER), neighbor mapping (NM), and unidirectional growth (UG) approaches, on the basis of simulated $\mathrm{F}_{2}$ data with different population sizes, missing genotype rates, and numbers of markers. Simulation results showed that the SDI method had a similar or higher percentage of correct linkage orders than the other three methods. This SDI algorithm was also applied to a real dataset and compared with the other three methods. The total linkage map distance $(\mathrm{cM})$ obtained by the SDI method $(148.08 \mathrm{cM})$ was smaller than the distance obtained by SER $(225.52 \mathrm{cM})$ and two published distances (150.11 $\mathrm{cM}$ and $150.38 \mathrm{cM})$. Since this SDI algorithm is stochastic, a more accurate linkage order can be quickly obtained by repeating this algorithm. Thus, this SDI method, which combines the advantages of accuracy and speed, is an important addition to the current linkage mapping toolkit for constructing improved linkage maps.

AMS 2000 SUbJeCt Classifications: Primary 60H30; secondary 60E15.

KEYWORdS AND PHRASES: Linkage mapping, Stochastic deletion-insertion algorithm.

\section{INTRODUCTION}

Molecular markers are essential for quantitative trait locus (QTL) mapping and gathering molecular genetic information for the improvement of plant and animal species. With the advance of technology, the number of loci examined in different chromosome regions is continuously growing, which has quickly increased the computational burden for constructing high quality linkage maps. Thus, a linkage mapping method with high accuracy and less computational burden is needed for ordering a large number of loci.

* Supported in part by the Agricultural Experiment Station at the South Dakota State University.

†Corresponding author.

$¥$ Supported in part by the National Institutes of Health Grant R01 DA025095.
Marker ordering was once considered a special case of the travelling salesman problem (TSP) [8], which is a classical non-deterministic polynomial-time (NP) complete problem $[16,11,4]$. There are two general types of approaches to tackling this problem. The first approach performs exhaustive searches to find the global optimal marker order solution. However, this approach is not practical when the number of linked loci is large because $n ! / 2$ distinct orders need to be evaluated for $n$ linked loci. Even for just 10 loci on one chromosome, there are 1,814, 400 possible orders. For many experiments, $n$ might vary from dozens to several hundreds. Thus, an exhaustive search is clearly computationally prohibited when the number of loci is greater than $30[9,10,14]$. The second approach uses approximation algorithms for determination of linkage order. These approximation algorithms for obtaining near-optimal marker order solutions are more practical for large-scale linkage mapping [8]. To date, several approximation algorithms for determination of linkage order have been proposed (i.e. $[1,13,15,7,3,2,14,12,10])$. All these linkage methods are based on the biological assumption that the true order of a set of linked loci has a minimum sum of adjacent recombination frequencies (SARF) or a maximum sum of adjacent LOD scores (SALOD).

Many approximation algorithms construct linkage maps sequentially; these algorithms start with only two markers and then add one of the remaining loci to the linkage map at a time until all loci are mapped. The advantage of the approximation mapping approaches is speed. However, since these algorithms are heuristic, the global optimal solution may not be reached if a two-point recombination frequency data set is not monotonic. Missing markers, crossover interference, and small population sizes can cause data nonmonotonicity. An alternative approximation algorithm is the evolutionary strategy (ES) algorithm [10], which starts with an initial set of many random linkage orders and eventually reaches an optimal linkage order through crossover and mutant operators. Thus, the final linkage order and the speed to reach this final solution greatly depend on the size of the initial set of linkage orders and the operators chosen.

Though a linkage order achieved by approximation methods may not be the global optimal solution if a dataset is not monotonic, a local linkage order and the global solution would have similar linkage orders. It is unlikely that a local linkage order obtained by an approximation method is 
Table 1. Four distinct linkage orders when a $4^{\text {th }}$ marker added to a partial linkage map with three markers

\begin{tabular}{llccc}
\hline \hline & \multicolumn{4}{c}{ Four possible linkage orders } \\
\hline $1:$ & $\mathbf{4} \rightarrow$ & $1 \rightarrow$ & $2 \rightarrow$ & 3 \\
$2:$ & $1 \rightarrow$ & $4 \rightarrow$ & $2 \rightarrow$ & 3 \\
$3:$ & $1 \rightarrow$ & $2 \rightarrow$ & $4 \rightarrow$ & 3 \\
$4:$ & $1 \rightarrow$ & $2 \rightarrow$ & $3 \rightarrow$ & 4 \\
\hline
\end{tabular}

completely different from the global optimal solution, implying a possibility that we can evolve a better/global order solution from some local optimal ones. Such a linkage mapping algorithm will improve accuracy compared to existing approximation methods while reducing the computational intensity relative to the exhaustive search algorithm. In this study, a stochastic deletion-insertion (SDI) algorithm, which minimizes SARF or maximizes SALOD values, is proposed. Various simulated $\mathrm{F}_{2}$ data were generated to evaluate the performance of this approach with different population sizes, numbers of markers, and missing genotype rates. A real data set was also used to demonstrate the benefit of this SDI algorithm.

\section{SDI ALGORITHM}

If an optimal solution for a partial linkage map [12] with three loci has been determined, denoted $1 \rightarrow 2 \rightarrow 3$, then an optimal linkage order with a new locus 4 on the same chromosome can be determined through comparing the four possible orders (Table 1). Thus, the linkage order with the locus 4 that minimizes the SARF or maximizes the SALOD value should be the optimal solution conditioning on the previous linkage order. This process can continue until all unmapped loci $n$ are added to the linkage map.

The SDI algorithm proceeds as follows:

- Step 1: Obtain the recombination frequency matrix for all possible pairs of $n$ loci on the same chromosome. If multiple linkage groups are found, a criterion is required to separate the different loci into more than one linkage group [17]. A corresponding distance matrix can be converted from the recombination frequency matrix using a mapping function (i.e., [5, 8]). Since the distance matrix and recombination frequency matrix used for linkage mapping result in the same marker order, without loss of generality, the recombination frequency matrix will be used throughout this study.

- Step 2: Generate an initial linkage order $L_{B}$ by using seriation (SER) [1], unidirectional growth (UG) [12], or neighbor mapping (NM) [3].

- Step 3: Deletion process:

- 3a: Generate a random number $s(1<s<n-1)$

- 3b: Randomly select $s$ loci from $n$ loci in linkage order $\mathrm{L}_{\mathrm{B}}$ to form a new sub linkage group $\mathrm{L}_{\mathrm{s}}$ without changing the order of these $s$ loci.
- Step 4: Insertion process: Randomly select one of unmapped $n-s$ loci and insert this locus into the linkage map $\mathrm{L}_{\mathrm{s}}$ with $(s+1)$ possible positions. Thus a new optimal linkage order with $(s+1)$ loci can be determined by comparing SARF. Repeat this process until all $n-s$ markers are mapped into $L_{s}$. If $L_{1}$ is better than $L_{B}$, then set $\mathrm{L}_{\mathrm{B}}=\mathrm{L}_{1}$.

- Step 5: Repeat Steps 3 to 4 for $N$ times. As an alternative, one can use simulated annealing or other proper approaches in this step.

- Step 6: Repeat Step 5 until no better solution is obtained for a given number of consecutive times (we used 10 times in this study).

If a recombination fraction matrix is monotonic, then the global optimal solution could be obtained at step 2. If the recombination fraction matrix is not monotonic, the linkage order obtained by the SDI method at each iteration may be a local optimal solution. Since the process of this deletion-insertion procedure is stochastic, the SDI method has a higher probability of reaching the global optimal solution by repeating Step 5 . It should be noted that a little larger $N$ which depends on the number of markers, genotype missing rate, population size, etc., can be employed (see Simulation Results for details) in Step 5 for a large number of linked loci because a desirable solution may not be reached due to possible immature convergence.

\section{SIMULATION SCENARIOS}

Since in reality, few real data sets are available with known marker orders, computer simulations are an alternative method for evaluating the performance of this SDI algorithm and several widely used approximation algorithms: SER [1], NM [3], and UG [12]. In this study we simulated an $\mathrm{F}_{2}$ population derived from a cross between two inbred lines because $\mathrm{F}_{2}$ populations that have more segregated types are a commonly used experimental design for linkage mapping studies. To better demonstrate the performance of the SDI algorithm, we carefully considered several factors that may contribute to data non-monotonicity: distance among linked loci, mapping population size, missing genotype rate, and recombination interference. To increase the possibility of generating a non-monotonic dataset, probability distributions for distance between adjacent markers were set as follows: $P(1<\mathrm{d}(\mathrm{cM})<5)=0.70, P(5<\mathrm{d}(\mathrm{cM})<10)=0.15$, $P(10<\mathrm{d}(\mathrm{cM})<30)=0.10$, and $P(30<\mathrm{d}(\mathrm{cM})<$ $40)=0.05$, with a uniform distribution within each of the four ranges. Data were simulated with six population sizes $(N=50,100,150,200,250$, or 300$)$, three numbers of co-dominant markers within the linkage group $(N=10,20$, or 50$)$, and three missing genotype rates $(0 \%, 10 \%$, or $15 \%)$. We also considered the presence of recombination interference. For simplicity of generating recombination interference, the recombination interference coefficient was randomly selected from the four possible values: 
1.0 (without inference), 0.0 (complete positive interference), 0.5 (partial positive interference), and 2.0 (negative interference). The recombination frequency between any two markers was calculated by the Expectation-Maximization (EM) algorithm [8]. The mean percentage of correct order (PCO) value [8], a probability that a locus is ordered correctly, was used to compare different mapping methods. All simulations were repeated 200 times for each of these configurations with a computer program in $\mathrm{C}++$ written by the authors of this paper. The significance of population size, marker number, missing genotype rate, and linkage mapping algorithm on PCO were estimated by one-way ANOVA by JMP 8.0 software (SAS Institute, Inc., Cary, NC).

\section{SIMULATION RESULTS}

\subsection{The impact of repetitions on PCO values for the SDI method}

Since an optimal linkage order obtained by the SDI approximation algorithm may be a local solution if a dataset is not monotonic, iterating the SDI algorithm is necessary to achieve a better solution. Therefore, it is helpful to determine the number of iterations sufficient to achieve a high PCO. Though we conducted various simulations for the impact of repetitions on PCO values, only the PCO values for each of six numbers of linked loci $(10,20,50,100,200$, and 500) from 1 to 100 repetitions over 20 simulated $\mathrm{F}_{2}$ data sets (10 with no missing rate and 10 with $10 \%$ missing rate, population size $=100)$ are reported in Figure 1. Our simulated results suggested that the PCO values remained stable after 20 repetitions (iterations) for most cases with 200 or fewer linked loci (Figure 1). The PCO values stabilized after only repeating 70 times for 500 linked loci. For a few cases (population sample size 50 with missing genotype rate $15 \%$ ), over 1,000 repetitions were needed before the PCO values stabilized (data not shown). Thus, the simulation results implied that this SDI algorithm could reach a desirable solution with a light computational burden. In our following simulations, five times $(N=5)$ in step 5 were used and 10 consecutive times in step 6 were employed until no better solution was obtained, thus there were at least 55 iterations for each simulated data set.

\subsection{Comparisons of PCO values among four mapping algorithm approaches}

The empirical PCO values for four approximation algorithms (SER, NM, UG, and SDI) were calculated by comparing simulated data with results from each of the approximation algorithms (Tables 2 and 3). The PCO values for the SDI algorithm were consistently equal to or higher than the other three approximation algorithms (Table 2). In addition, the SDI algorithm was robust for small population sizes and maintained a high PCO (>90\%) when the missing genotype data rate was high $(15 \%)$ and the population size was $\geq 100$.

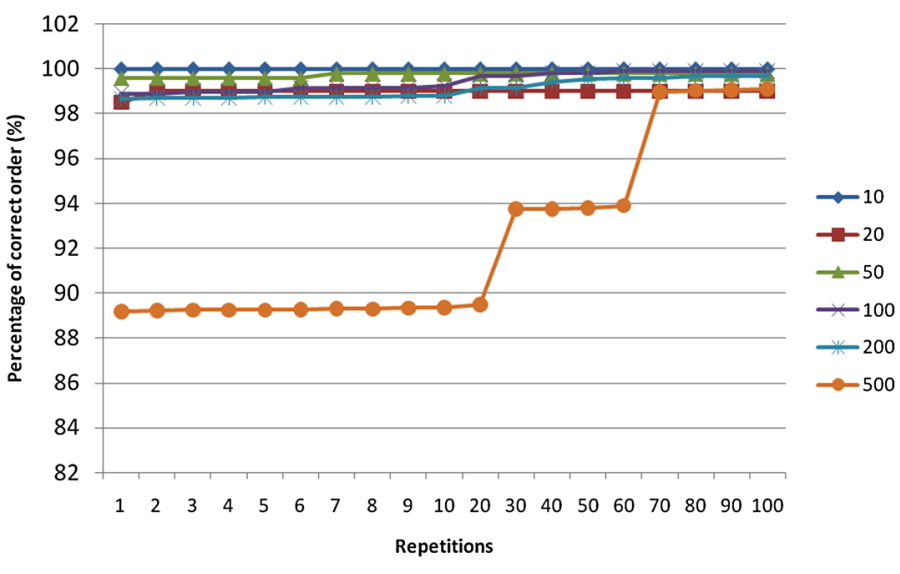

Figure 1. The impact of repetitions on percentage of correct order (PCO) over $20 \mathrm{~F}_{2}$ simulated data sets (10 data sets with no missing data rate and 10 with $10 \%$ missing rates) for each of six numbers of linked loci

$(10,20,50,100,200$, and 500).

Therefore, our results suggest that the SDI method was the best among these four algorithms. On average, the NM algorithm was the poorest among the four methods and was sensitive to small population sizes, large marker numbers, and high missing genotype rates. The other two algorithms (SER and UG) performed nearly equally well in our simulation study: PCOs were often higher than the NM but lower than the SDI.

As expected, PCO increased with larger population sizes, smaller number of markers, and lower missing genotype rates (Table 2). When the missing genotype rate was $0 \%$, a desirable mapping power $(\geq 95 \%)$ could be achieved by SER, UG, and SDI algorithms when population size was $\geq 100$. When the missing genotype rate was $10 \%$ or $15 \%$, a desirable mapping power $(\geq 95 \%)$ could be achieved for SER, UG, and SDI when population size $\geq 200$. The NM algorithm required a population size $\geq 200$ to consistently achieve a $\mathrm{PCO} \geq 95 \%$ when the missing genotype rate was $0 \%$. When the missing genotype rate was $15 \%$, the NM algorithm did not achieve a PCO $\geq 95 \%$ under all simulated situations.

Population size, linkage mapping algorithm, and missing genotype rate were significantly associated with PCO $(P<0.05)$ (Table 3). Population size explained the largest amount of variation in PCO $\left(\mathrm{R}^{2}=0.478\right)$ when estimating marker order with our simulated dataset. The PCO was $>95 \%$ when the population size was 150 or greater. Number of markers was not significantly associated with PCO $(P=0.128)$, although the PCO decreased as the number of markers increased (in general, as the number of markers increases, both the chance to cause non-monotonicity of the recombination frequency matrix and the solution space will increase, resulting in a higher probability to be trapped by a local solution and thus a reduced $\mathrm{PCO}$ ). 
Table 2. The percentage of correct orders (PCO, \%) obtained by four algorithms for six population sizes (PS), three missing genotype rates (MS, \%) and different numbers of markers (MN) on one chromosome

\begin{tabular}{cc|ccc|ccc|ccc|ccc}
\hline \hline MN & PS & \multicolumn{3}{|c|}{ SER $^{1}$} & \multicolumn{3}{c|}{ UG } & \multicolumn{3}{c}{ NM } & \multicolumn{3}{c}{ SDI } \\
\hline & & $0 \%$ & $10 \%$ & $15 \%$ & $0 \%$ & $10 \%$ & $15 \%$ & $0 \%$ & $10 \%$ & $15 \%$ & $0 \%$ & $10 \%$ & $15 \%$ \\
\hline 10 & 50 & 94.50 & 87.90 & 83.05 & 93.65 & 87.90 & 81.60 & 82.10 & 70.55 & 63.90 & 95.25 & 91.30 & 87.35 \\
& 100 & 99.05 & 95.10 & 95.70 & 98.30 & 95.95 & 94.30 & 89.95 & 81.25 & 79.00 & 99.25 & 96.90 & 97.80 \\
& 150 & 98.60 & 97.70 & 97.00 & 98.40 & 97.05 & 97.10 & 94.05 & 88.05 & 83.00 & 98.80 & 98.00 & 97.35 \\
& 200 & 99.90 & 99.55 & 99.00 & 99.90 & 99.50 & 99.30 & 96.70 & 90.35 & 89.45 & 99.90 & 99.75 & 99.50 \\
& 250 & 99.50 & 99.35 & 99.45 & 99.40 & 99.55 & 99.00 & 98.00 & 93.00 & 90.70 & 99.70 & 99.55 & 99.50 \\
& 300 & 100.00 & 98.90 & 100.00 & 99.20 & 99.15 & 99.10 & 98.30 & 92.55 & 92.35 & 100.00 & 99.75 & 99.65 \\
20 & 50 & 92.77 & 83.25 & 78.57 & 93.53 & 81.40 & 81.33 & 84.95 & 66.85 & 62.05 & 95.10 & 89.00 & 86.30 \\
& 100 & 94.35 & 95.38 & 94.33 & 94.23 & 94.85 & 94.20 & 92.80 & 84.67 & 84.17 & 96.58 & 97.42 & 97.97 \\
& 150 & 97.78 & 97.02 & 97.15 & 97.08 & 95.67 & 96.53 & 96.03 & 90.95 & 88.80 & 97.85 & 98.30 & 98.20 \\
& 200 & 97.80 & 98.17 & 96.58 & 96.78 & 95.58 & 95.53 & 98.30 & 95.17 & 89.42 & 98.85 & 99.10 & 97.32 \\
& 250 & 99.20 & 98.28 & 96.05 & 98.35 & 97.42 & 94.53 & 98.63 & 96.10 & 91.95 & 99.25 & 98.42 & 96.63 \\
& 300 & 99.30 & 98.90 & 97.97 & 97.90 & 98.08 & 97.65 & 98.72 & 96.15 & 94.63 & 99.30 & 98.90 & 98.58 \\
50 & 50 & 89.49 & 76.52 & 67.09 & 88.50 & 78.20 & 71.06 & 85.22 & 61.18 & 51.31 & 92.81 & 87.62 & 79.15 \\
& 100 & 96.08 & 93.28 & 89.46 & 95.63 & 91.91 & 85.47 & 95.20 & 81.17 & 72.44 & 96.45 & 96.66 & 93.65 \\
& 150 & 98.57 & 97.52 & 94.77 & 95.17 & 96.60 & 90.50 & 98.30 & 92.38 & 83.70 & 98.75 & 99.21 & 96.17 \\
& 200 & 98.88 & 97.03 & 98.14 & 97.00 & 92.29 & 95.27 & 98.95 & 94.19 & 91.68 & 99.16 & 97.78 & 98.51 \\
& 250 & 96.34 & 97.20 & 95.98 & 94.98 & 92.98 & 92.93 & 95.91 & 95.30 & 91.96 & 96.84 & 97.21 & 96.34 \\
& 300 & 98.90 & 98.63 & 97.29 & 96.83 & 96.66 & 94.13 & 98.94 & 98.07 & 93.65 & 99.02 & 98.67 & 97.79 \\
\hline
\end{tabular}

${ }^{1} \mathrm{SER}=$ seriation, $\mathrm{UG}=$ unidirectional growth, $\mathrm{NM}=$ neighbor mapping, and SDI = stochastic deletion-insertion.

Table 3. Effects of population size (PS), marker number $(M N)$, missing genotype rates (MS, \%), and linkage mapping algorithm (LMA) on percent correct order $(P C O, \%)^{1}$

\begin{tabular}{|c|c|c|c|c|c|}
\hline Effect & Level & $\begin{array}{c}\text { Mean PCO, } \\
\%\end{array}$ & F-ratio & $\mathrm{P}$-value & $\operatorname{Adj} R^{2}$ \\
\hline PS & $\begin{array}{c}50 \\
100 \\
150 \\
200 \\
250 \\
300\end{array}$ & $\begin{array}{l}81.73 \\
92.53 \\
95.50 \\
95.90 \\
96.82 \\
97.88\end{array}$ & 40.35 & $<0.0001$ & 0.478 \\
\hline $\mathrm{MN}$ & $\begin{array}{l}10 \\
20 \\
50\end{array}$ & $\begin{array}{l}94.65 \\
93.98 \\
92.06\end{array}$ & 2.07 & 0.128 & 0.010 \\
\hline MS, \% & $\begin{array}{c}0 \\
10 \\
15\end{array}$ & $\begin{array}{l}96.52 \\
93.22 \\
90.96\end{array}$ & 9.61 & 0.0001 & 0.074 \\
\hline $\mathrm{LMA}^{2}$ & $\begin{array}{c}\text { NM } \\
\text { SDI } \\
\text { SER } \\
\text { UG }\end{array}$ & $\begin{array}{l}88.21 \\
96.74 \\
95.15 \\
94.17\end{array}$ & 13.98 & $<0.0001$ & 0.153 \\
\hline
\end{tabular}

$\overline{{ }^{1} \text { F-ratios, P-values, and adjusted } R^{2} \text { calculated individually for }}$ each effect by one-way ANOVA.

${ }^{2} \mathrm{SER}=$ seriation, $\mathrm{UG}=$ unidirectional growth, $\mathrm{NM}=$ neighbor mapping, and SDI = stochastic deletion-insertion.

384 J. Wu, X.-Y. Lou and M. Gonda
The NM algorithm on average resulted in the lowest PCO (88.21\%). The SDI algorithm resulted in the highest PCO (96.74\%), although both SER and UG algorithms had PCOs $(95.15 \%$ and $94.17 \%$, respectively) similar to the SDI.

\section{A REAL DATASET}

To evaluate the performance of the SDI algorithm, we applied SDI to a real dataset of 26 loci on barley chromosome I from the North American Barley Genome Mapping project (see Liu 1998, [8], p. 288). Though the number of linked loci was 26 , there are two reasons we chose this dataset: (1) the recombination matrix was not monotonic because different approximation methods generated different linkage orders and (2) two different linkage orders have been published using this dataset $[8,12]$. The linkage orders obtained by the SER and SDI methods in this paper were compared to the two published orders $[8,12]$. The genetic distances presented in this paper were calculated based on Haldane's mapping function [5], so the total distances listed in Table 4 for the two published linkage orders are slightly different from the published distances $[8,12]$. Even though the different mapping functions may slightly result in different genetic distances, the marker orders from the different mapping functions still remained the same. Results showed that the total distances obtained by the UG algorithm [12] and the combination of Simulated Annealing (SA) and Branch and Bound (BB) algorithms used by Liu [8] were very close to each other (150.38 cM vs $150.11 \mathrm{cM}$, respectively) (Table 4). The SER algorithm generated the largest genetic distance $(225.52 \mathrm{cM})$, while the SDI algorithm generated the shortest 
Table 4. Marker orders and their genetic distances (cM) obtained from different mapping algorithms

\begin{tabular}{|c|c|c|c|c|c|c|c|}
\hline \multicolumn{2}{|c|}{$\mathrm{Liu}^{1}$} & \multicolumn{2}{|c|}{$\operatorname{Tan} \& \mathrm{Fu}$} & \multicolumn{2}{|c|}{ SER } & \multicolumn{2}{|c|}{$\overline{~ S D I}$} \\
\hline Order & Dist & Order & Dist & Order & Dist & Order & Dist \\
\hline $\begin{array}{ll}2 & 1\end{array}$ & 0.00 & 1 & 0.00 & 1 & 0.00 & 1 & 0.00 \\
\hline 2 & 12.65 & 2 & 12.65 & 2 & 12.65 & 2 & 12.65 \\
\hline 3 & 4.80 & 3 & 4.80 & 3 & 4.80 & 3 & 4.80 \\
\hline 4 & 15.52 & 4 & 15.52 & 5 & 16.47 & 5 & 16.47 \\
\hline 5 & 3.94 & 5 & 3.94 & 4 & 3.94 & 4 & 3.94 \\
\hline 6 & 11.55 & 7 & 13.03 & 7 & 11.61 & 7 & 11.61 \\
\hline 7 & 0.76 & 6 & 0.76 & 6 & 0.76 & 6 & 0.76 \\
\hline 8 & 8.68 & 9 & 5.05 & 8 & 7.67 & 9 & 5.05 \\
\hline 9 & 0.77 & 8 & 0.77 & 9 & 0.77 & 8 & 0.77 \\
\hline 10 & 1.52 & 10 & 2.11 & 10 & 1.52 & 10 & 2.11 \\
\hline 11 & 2.93 & 11 & 2.93 & 11 & 2.93 & 11 & 2.93 \\
\hline 12 & 2.25 & 12 & 2.25 & 12 & 2.25 & 12 & 2.25 \\
\hline 13 & 5.22 & 14 & 2.27 & 14 & 2.27 & 13 & 5.22 \\
\hline 14 & 4.51 & 13 & 4.51 & 15 & 3.10 & 14 & 4.51 \\
\hline 15 & 3.10 & 15 & 7.89 & 16 & 8.42 & 15 & 3.10 \\
\hline 16 & 8.42 & 16 & 8.42 & 17 & 13.29 & 16 & 8.42 \\
\hline 17 & 13.29 & 17 & 13.29 & 18 & 6.99 & 17 & 13.29 \\
\hline 18 & 6.99 & 18 & 6.99 & 19 & 21.89 & 18 & 6.99 \\
\hline 19 & 21.89 & 19 & 21.89 & 20 & 3.54 & 19 & 21.89 \\
\hline 20 & 3.54 & 20 & 3.54 & 21 & 2.21 & 20 & 3.54 \\
\hline 21 & 2.21 & 21 & 2.21 & 22 & 0.00 & 21 & 2.21 \\
\hline 22 & 0.00 & 22 & 0.00 & 24 & 0.73 & 22 & 0.00 \\
\hline 23 & 2.17 & 23 & 2.17 & 23 & 1.48 & 23 & 2.17 \\
\hline 24 & 1.48 & 24 & 1.48 & 25 & 8.34 & 24 & 1.48 \\
\hline 25 & 3.70 & 25 & 3.70 & 26 & 8.21 & 25 & 3.70 \\
\hline 26 & 8.21 & 26 & 8.21 & 13 & 79.68 & 26 & 8.21 \\
\hline 3 & 150.11 & & 150.38 & & 225.52 & & 148.08 \\
\hline
\end{tabular}

${ }^{1}$ Liu = the combination of Simulated Annealing (SA) and Branch and Bound (BB) algorithms, Tan \& Fu = unidirectional growth $(\mathrm{UG}), \mathrm{SER}=$ seriation, and SDI = stochastic deletion-insertion.

${ }^{2}$ Marker codes for $1 \sim 26$ and the linkage order were reported by Liu (1998); the linkage order obtained by the UG method was reported by Tan and Fu (2006); and linkage orders by the other two methods were reported by this paper.

${ }^{3}$ The values in the bottom line represent the genetic distance obtained by Haldane's function.

distance $(148.08 \mathrm{cM})$ by updating the linkage order obtained by the SER method with a small number of iterations.

\section{DISCUSSION}

As pointed out earlier, the exhaustive search algorithm can be applied to find the global optimal solution only when the number of linked loci is small. The evolutionary strategy (ES) algorithm can have the potential to find the correct linkage order but it is computationally intensive and the final solution is dependent on many factors such as the cross operator, mutation operator, and population size. On the other hand, many approximation algorithms like NM, SER, and UG have the advantage of speed but may not find the global optimal linkage order when the data is nonmonotonic. If a two-point dataset for linkage mapping is monotonic, all these approximation algorithms can reach the global optimal linkage order. However, the recombination frequency matrix is calculated from a sample, which could be influenced by many factors like sample size, linkage distance among markers, presence of crossover interference, and missing genotypes [9, 10].

Through partially updating linkage orders obtained by approximation methods, the SDI algorithm successfully improved mapping accuracy. The first advantage of this SDI algorithm is the high power in recovering the true map. Our simulations showed that the SDI method was the best among four methods to resolve true linkage orders. The application to an experimental data set [8] also showed that the SDI method could find the linkage order with the shortest linkage distance. The second advantage of the SDI method is to balance the accuracy and computing speed well. Compared to several approximate algorithms such as SER, UG and UM, the SDI algorithm only takes a few additional iterations and minor extra computational time to reach an improved solution; however, it greatly reduces computational intensity as compared to the exhaustive search algorithm.

Two significant differences exist between the SDI method and other approximation methods. The first difference is 
that many approximation methods add one remaining locus onto one of two ends of a partial linkage order at each step. Therefore, these methods are hill-climbing approaches. The SDI method, however, allows an unmapped locus to be added onto any position of a partial linkage order at each step, increasing the chance of obtaining a more accurate linkage map. The second difference is that previous approximation methods can obtain only one solution; whereas, the SDI algorithm generates more than one optimal solution, leading to improved power for finding a more accurate solution. In practice, researchers can choose the best linkage order from several approximation methods as an initial order. Then, the SDI algorithm can be employed to update and improve this linkage order as more data are collected. Therefore, this SDI algorithm should be an important supplement to the current tools to improve linkage mapping power.

Linkage power depends on estimates of recombination frequencies. Many factors such as linkage distance, population size, missing genotype rate, and crossover interference can lead to biased estimation and thus cause non-monotonicity of the recombination frequency matrix [9]. These four factors were considered in our simulation study to increase the possibility of non-monotonic data. It appeared that the SDI algorithm was robust to non-monotonic data in recovering true linkage maps after a few iterations; however, we observed that a large number of iterations (more than 1,000) were needed to resolve true linkage orders for a few cases (small population sizes and high missing genotype rates). This observation suggests that a large $N$ in step 5 will be needed to achieve a more accurate linkage order for a large number of linked loci in a small population with a high missing genotype rate. We also observed that resolving powers decreased when the number of iterations for the SDI method increased in several cases where the populations size was small (50) with high missing genotype rates (10\% and above). This result suggests that small population sizes and high missing genotype rates should not be recommended when constructing large-scale linkage maps.

The types of markers in an $\mathrm{F}_{2}$ population are related to the mapping power as well [6, 8, 17, 9]. Dominant markers act differently under conditions of couplingphase and repulsion-phase. When all dominant markers were in coupling-phase, the proportion of dominant and co-dominant markers had little impact on mapping power $[9,17]$. However, the repulsion-phase dominant markers often caused biased estimations and thus low mapping power $[8,17]$. Hence, the direct use of minimum SARF or maximum SALOD values may not work efficiently when markers are in repulsion-phase. Knapp et al. [6, 9] suggested that two complimentary linkage orders, each including co-dominant markers, can be constructed. An alternative methodology is to use the SDI method to merge two complimentary maps into one. Further investigation is needed to improve the accuracy of marker order and estimating linkage distance.

\section{CONCLUSIONS}

A stochastic deletion-insertion (SDI) algorithm for constructing large-scale linkage maps was proposed in this study. The mapping power of this SDI algorithm was evaluated along with three published approximation approaches: SER, NM, UG, on the basis of simulated $\mathrm{F}_{2}$ data with different population sizes, missing genotype rates, and numbers of linked loci. Simulation results showed that the SDI method had similar or higher PCO values than the other three methods. The NM algorithm on average resulted in the lowest PCO (88.21\%). The SDI algorithm resulted in the highest PCO (96.74\%). Both SER and UG algorithms had PCOs (95.15\% and $94.17 \%$, respectively) less than the SDI. The SDI algorithm was also applied to a real dataset and compared with the other three methods. The total linkage map distance obtained by the SDI method was smaller than the distance obtained by SER and two published distances. Since this SDI algorithm is stochastic and progressive-iterative, it increases the possibility to obtain a more accurate linkage order. Thus, this SDI method, which combines the advantages of accuracy and speed, is an important addition to the current linkage mapping toolkit for constructing dense linkage maps.

\section{ACKNOWLEDGEMENTS}

The authors would like to thank the two reviewers for their helpful comments that have helped improve this paper. We are also grateful to Krishna Bondalapati for her great help reformatting this paper.

Received 1 June 2010

\section{REFERENCES}

[1] Buetow, K. H. and Chakravarti, A. (1987). Multipoint gene mapping using seriation. I. General methods. American Journal of Human Genetics 41 180-188.

[2] Crane, C. F. and Crane, Y. M. (2005). A nearest-neighboringend algorithm for genetic mapping. Bioinformatics 21 1579-1591.

[3] Ellis, T. H. N. (1997). Neighbour mapping as a method for ordering genetic markers. Genetical Research 69 35-43.

[4] FALK, C. T. (1992). Preliminary ordering of multiple linked loci using pairwise linkage data. Genetic Epidemiology 9 367-375.

[5] Haldane, J. B. S. (1919). The combination of linkage values and the calculation of distances between the loci of linked factors. Journal of Genetics 8 299-309.

[6] Knapp, S. J., Holloway, J. L., Bridges, W. C. and Liu, B. H. (1995). Mapping dominant markers using F2 mating. Theoretical and Applied Genetics 91 74-81.

[7] Lathrop, G. M., Lalouel, J. M., Julier, C. and Ott, J. (1985). Multilocus linkage analysis in humans: detection of linkage and estimation of recombination. American Journal of Human Genetics 37 482-498.

[8] Liu, B. (1998). Statistical Genomics: Linkage, Mapping, and QTL Analysis. CRC Press LLC.

[9] Mester, D. I., Ronin, Y., Hu, Y., Peng, J., Nevo, E. and KoROL, A. (2003a). Efficient multipoint mapping: making use of dominant repulsion-phase markers. Theoretical Applied Genetics 107 1102-1112. 
[10] Mester, D. I., Ronin, Y., Mlinkov, D., Nevo, E. and Korol, A. (2003b). Constructing large scale genetic maps using an evolutionary strategy algorithm. Genetics 165 2269-2282.

[11] Olson, J. M. and Boennke, M. (1990). Monte Carlo comparison of preliminary methods for ordering multiple genetic loci. American Journal of Human Genetics 47 470-482.

[12] TAN, Y. and Fu, Y. (2006). A novel method for estimating linkage maps. Genetics 173 2383-2390.

[13] Thompson, E. (1984). Information gain in joint linkage analysis. IMA Journal of Mathematics Applied in Medicine and Biology 1 $31-49$.

[14] Van Os, H., Stam, P., Visser, R. G. F. and Van Eck, H. J. (2005). RECORD: a novel method for ordering loci on a genetic linkage map. Theoretical and Applied Genetics 112 30-40.

[15] WeEks, D. and LANGe, K. (1987). Preliminary ranking procedures for multilocus ordering. Genomics 1 236-242.

[16] Wilson SR, M. (1988). A major simplification in the preliminary ordering of linked loci. Genetic Epidemiology 5 75-80.

[17] Wu, J., Jenkins, J., Zhu, J., McCarty, J. and Watson, C. (2003). Monte Carlo simulations on marker grouping and ordering. Theoretical and Applied Genetics 107 568-573.

\section{Jixiang $\mathrm{Wu}$}

Plant Science Department

South Dakota State University

Box 2140C, Brookings

SD 57007

USA

E-mail address: jixiang.wu@sdstate.edu
Xiang-Yang Lou

Section on Statistical Genetics

Department of Biostatistics

University of Alabama at Birmingham

Royals Public Health Building, Suite 414

1665 University Boulevard

Birmingham

Alabama 35294

USA

E-mail address: XLou@ms.soph.uab.edu

Michael Gonda

Animal and Range Science Department

South Dakota State University

Box 2170, Brookings

SD 57007

USA

E-mail address: michael.gonda@sdstate.edu 